\title{
Revisiting Stereotomic Principles in Contemporary AEC Practice
}

\section{Edoardo Tibuzzi ${ }^{1}$ (D)}

Published online: 12 September 2018

(c) Kim Williams Books, Turin 2018

\begin{abstract}
The past 15 years have been characterised by constant technological and digital innovations, and, as a consequence, computer science has evolved past its academic and elitist status to become the common language adopted by professionals in many fields, revolutionising the methods and tools, and even creating new industries and markets. This shift permeated the architectural and construction industry, dramatically influencing the form and function of modern buildings. Stereotomy, one of the most fascinating and complex techniques in the history of pre-modern architecture, captures best the potential of being able to use digital design to integrate architectural form, structural integrity and environmental performance in a single multi-performative "skin". The intention of the following text is to highlight some application of new digital technologies in real life projects that fundamentally have a close link to the traditional stereotomic principles of "solid cutting" (Fallacara and Barberio in Handbook of research on form and morphogenesis in modern architectural contexts. IGI Global, Hershey, 2017).
\end{abstract}

Keywords Stereotomy $\cdot$ Digital stereotomy $\cdot$ Topological stereotomy $\cdot$ Robotic fabrication · Computational design · Digital fabrication · Stone architecture · Interlocking materials

\section{Introduction}

The past 15 years have been characterised by constant technological and digital innovations, and, as a consequence, computer science has evolved past its academic and elitist status to become the common language adopted by professionals in many fields, revolutionising the methods and tools, and even creating new industries and markets. This shift permeated the architectural and construction industry, dramatically influencing the form and function of modern buildings. It also

Edoardo Tibuzzi

edoardo.tibuzzi@akt-uk.com

1 AKT II, 1 Old Street Yard EC1Y 8AF, London, UK

Birkhäuser 
contributed to re-aligning the areas of architecture, engineering, environmental design, fabrication and construction that had, over the years, become fragmented and disconnected.

The ability to access vast calculation power in relatively small computers, and to connect geometry, material and structural performance and detailed modelling, saw the rediscovery of interesting theories that were applied in architecture at the beginning of the century or even earlier. Graphic statics, catenary shapes and form finding became fashionable again; the addition of the much respected prefix "computational" almost elevates these concepts and distinguishes them from the more analogical predecessors. Stereotomy, one of the most fascinating and complex techniques in the history of pre-modern architecture, captures best the potential of being able to use digital design to integrate architectural form, structural integrity and environmental performance in a single multi-performative "skin".

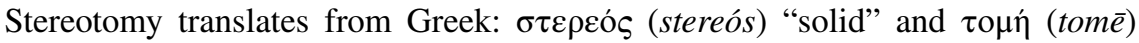
"cut". It is the art and science of cutting three-dimensional solids into particular shapes (Fallacara 2007: 22). As its application dates back to the 15th century, and even for contemporary experiments (Colella 2017), stereotomy is often associated to materials such as stone or wood, which is cut to be assembled into complex structures. Due to the complexity of the fabrication of these structures, descriptive geometry was implemented to represent in two dimensions the convoluted threedimensional setting out of the pieces. The interesting aspect of all stereotomic applications is the deep interconnection between materiality, behaviour, fabricator expertise and complex techniques. An interesting parallel starts to appear between the application of stereotomy and some new construction methods that are emerging today. In this perspective, it is important to underline that stereotomy today is no longer a technical knowledge related only to stone architecture (Fallacara and Barberio 2018). In the field of digital stereotomy, despite the relationship between stereotomy and stone (Fallacara 2012; Block et al. 2017; Fallacara and Barberio 2018), the last decade has seen other materials employed in effective ways. Examples include the use of plywood (Clifford and McGee 2014), cork (Merritt and Varela 2014), autoclaved aerated concrete (Clifford et al. 2016) and other cast materials (Varela and Sousa 2018), polystyrene (McGee et al. 2013) and plastics (Diles 2018).

The intention of the following text is to highlight some application of new digital technologies in real life projects that fundamentally have a close link to the traditional stereotomic principles of "solid cutting".

These projects developed at AKT II in London (Kara and Bosia 2016) share commonalities with creating structure by carving, cutting and interlocking different materials with different shapes, such as interlocking reciprocal configurations (CocaCola Beatbox Façade), sphere packing and mild steel cnc cutting (Odin Installation), soap-bubble, minimal surface and weaving (Tangling at the Architecture Foundation), stress transferring structural steel, semi-monocoque structures (Neuron Pod at Centre of the Cell). 


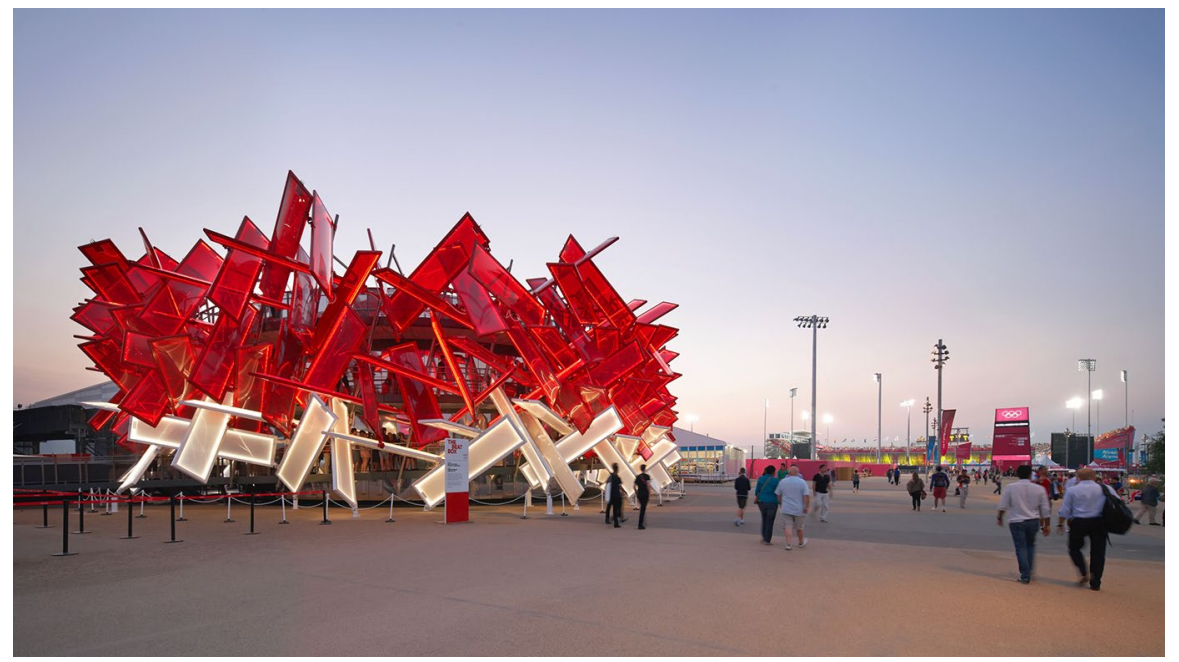

Fig. 1 Coca Cola Beatbox

\section{Coca-Cola Beatbox Façade, London 2012}

Based on a reciprocal triangular tiling of prismatic units, the façade of the CocaCola (see Fig. 1) pavilion is a rigid, self-supporting structure of $5 \times 1 \mathrm{~m}$ ETFE pillows, acting as interlocking air beams to form a cylindrical enclosure. This wraps around two spiralling ramps and an internal drum of a building to create a responsive, animated and musical three-dimensional curtain, giving the pavilion its name, Beatbox. When the design phase commenced on the competition-winning sketch of the pavilion, the façade had not been resolved geometrically. The over 300 pillows hovered in space in a seemingly random chaotic scatter, and each would have required a series of individual brackets supporting it back to the main frame of the building; an expensive and logistically unmanageable proposition. The countdown to the opening ceremony and the start of the Olympic Games had started and time was of the essence. On the other hand, the client was pushing for a simpler twodimensional pattern, more in tune with their simple, sleek branding.

The solution was to pack the pillows tight, into a more organised geometry, generating a sufficient number of connections at the points of tangency, such that the structure could become a rigid, self-supporting system. We studied flocking patterns and simulated magnetic attractors to produce packing arrangements, but the geometry of the connections varied, producing over 500 different conditions and making the system unbuildable within the budget and time constraints.

Combining some interesting studies on three-dimensional tiling with research done on reciprocal interweaving patterns, a proposal was made to the lead designers, Asif and Pernilla. While the tiling seemed to produce the perfect setting out for a modular and easily buildable façade, where each pillow and connection was geometrically identical, its reciprocal nature insured structural continuity and load transfer directly back to the building mainframe and to the 
ground. The system was also three-dimensional and spatial, offering architectural and environmental opportunities (see Fig. 2).

This reciprocal tiling became the façade and structure of the building envelope but also a three-dimensional interactive journey, through which people could circulate up to a viewing platform at the top of the building. As the name Beatbox suggests, the façade is a musical instrument, where each pillow is equipped with a touch sensor that causes the pillows to emit sounds and light pulses when visitors come in contact with it. As the pillows completely define the space and experience of the visitors, the tiling geometry had to be sufficiently flexible in its configuration to form entrances and views onto the surrounding park, as well shading of direct sunlight and protecting from the elements. This was achieved without breaking the connectivity of the reciprocal tiling by sliding or flipping each pillow along its axis or removing a pillow from the module, always within the organised prismatic grid. Although based on a simple geometric principle, the overall three-dimensional effect of the façade is a complex one, almost chaotic but nevertheless structured, rhythmic, almost musical in its ordered geometric sequence. With an A:B:A rhythm, the pattern seems to be setting the beat of the moving crowds that wiz through its spiralling ramps.

From a practical point of view, the geometric reciprocal tiling allowed the description of the whole façade with just a handful of drawings, automatically generated from a parametric model. From these drawings, identical pillows and connections where prefabricated and systematically and efficiently assembled on site. Groups of three pillows at a time could be preassembled in self-stable assemblies or larger façade panels and consistently fixed to the main frame of the building, almost as a standard curtain wall system, to reduce the amount of work on site. As the project had to follow a challenging procurement schedule to be completed on time, the design period was compressed to weeks rather than months. This was not sufficient to resolve all the specific conditions required, such as entrances and openings, as well as coordination with other elements of the architecture and the specific site constraints, such as varying ground levels. Again the geometric tiling was crucial to predefining the setting out of the façade framework, while allowing local sliding and flipping of the pillows at a later stage, without impacting on fabrication and construction (see Fig. 3).
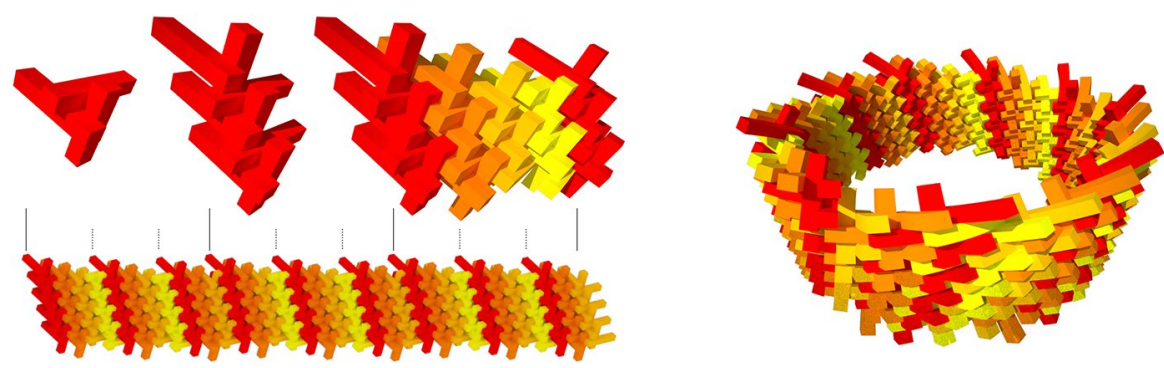

Fig. 2 Reciprocal Tiling Pattern 

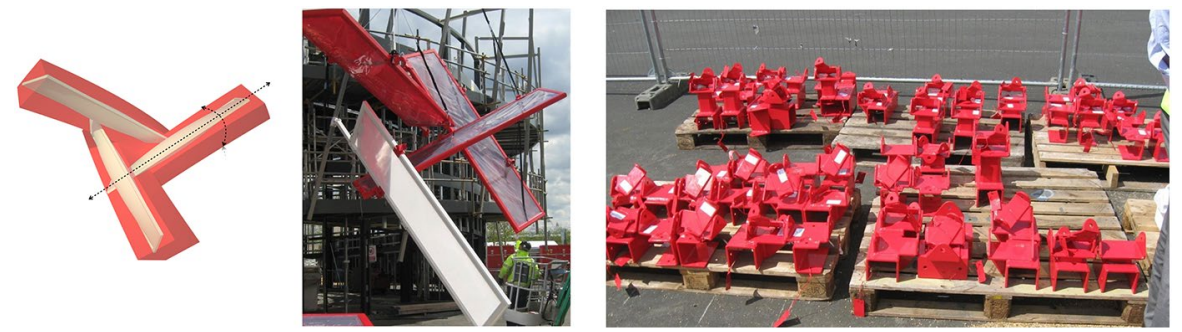

Fig. 3 Reciprocal frame, installation and custom bracket system

In essence, the rigor of the geometric tiling enabled the creation of a complex three-dimensional pattern, ensuring its structural continuity and its complete modularity, and yet allowed enough freedom to modulate its specific local conditions in a systematic, parametric and automated way. Furthermore, the underlying orders of its geometry informed a new emerging aesthetic that embraces complexity and richness within its embedded rhythms and patterns, and creates a piece which subtly borders between random chaos and structured order. Completed for the London 2012 Olympics, the pavilion was open to the public for the entire duration.

\section{Odin Installation, Colgate University, Hamilton, NY}

This project was born of collaboration between the sciences and the arts. Artist DeWitt Godfrey's formal vocabulary is characterised by the packing or stacking of circles in space. In a sort of reverse process from stereotomy, the solid is 'found' by filling the space using circle packing algorithms to generate the form.

The frustums (Corten steel coils) (see Fig. 4) were envisaged to be arranged on an underlying 'virtual' surface and connected to one another through steel bolts to create an interlocked structural surface. A virtual tool was developed for the entire project to perform five crucial operations: manipulate the generative surface, achieve a variation of density and radii of circles, retrieve a responsive structural feedback, be able to control the circle thickness and automatically produce fabrication drawings (see Fig. 5).

The initial surface was digitally moulded by the artist using the virtual tool, which allowed several operations to simulate the process of modelling with clay. Once the surface was defined, the following step was to input the number of circles and their radii variation, along with a preferred distribution (using a simple percentage value). These parameters were explored and set by the artist in the predefined algorithm in order for him to explore different variations of circle arrangements along the surface. The virtual tool would also allow the designer to fix certain circles in specific positions along the surface to define potential ingress zones or simply to constrain specific areas to the site. 


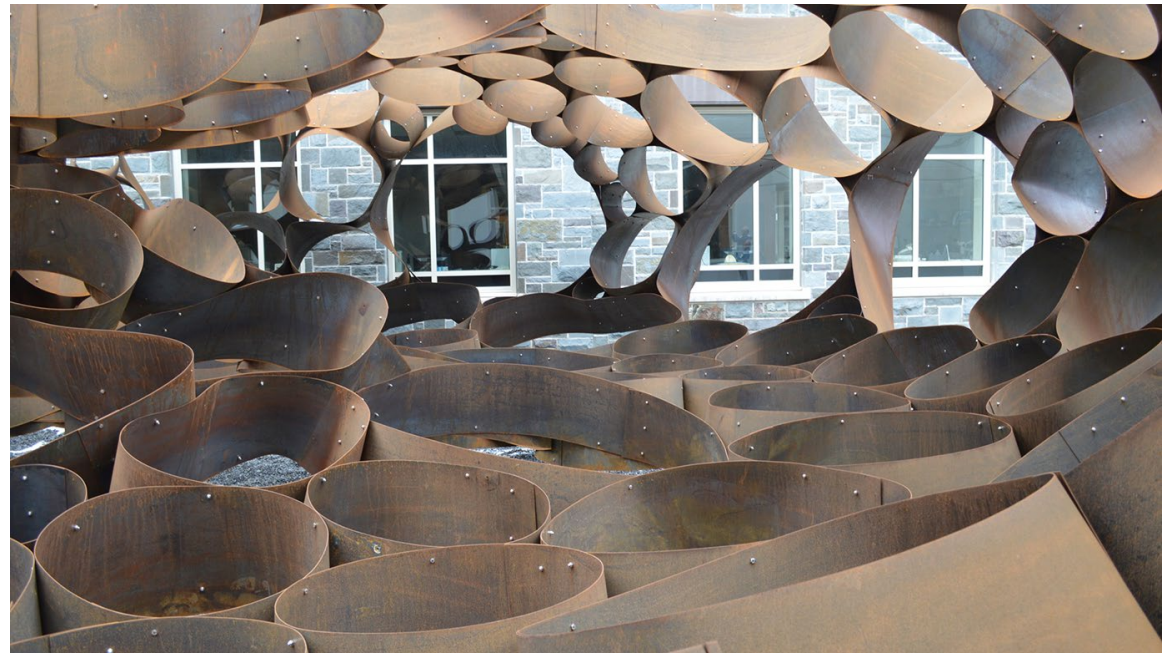

Fig. 4 Odin

At this point the circle pack algorithm would be initiated with the result being a surface filled with an arrangement of circles such that no two overlap and some (or all) of them are mutually tangent. The resulting geometric model, consisting of curves oriented on a NURBS surface, was then developed further to create the final frustums by extruding the circles into conical sections, making sure every interface would keep the original tangency and that every circle would share a unique linear intersection with its neighbour (see Fig. 6).

By interfacing directly with a FEM analysis software, the geometry was seamlessly analysed under the given loading constraints and specific options assessed. The results were instantly accessible in order to identify the peak stresses, overall deflections and local connection forces. This allowed the design team to inform the larger diameter circles and increase the plate thickness to reduce deformations and stresses. Finally, once the process had gone through several iteration of form finding, structural optimisation and fine-tuning, the last part of the virtual tool would take the input of the finalised frustums and perform an automatic unroll, creating a set of fabrication drawings including the dimensional information
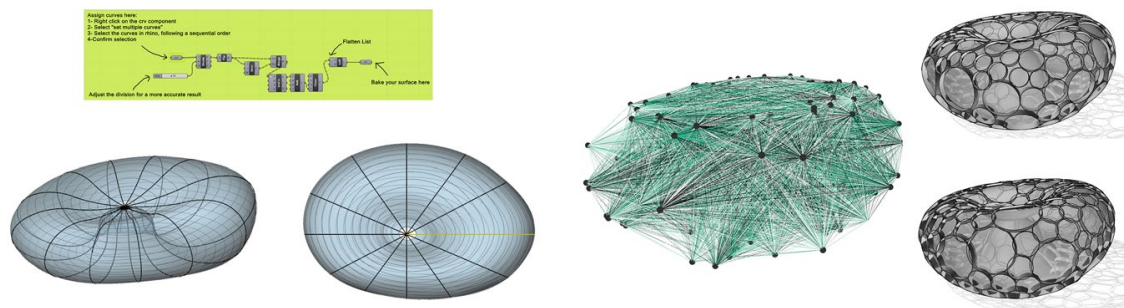

Fig. 5 Generative surface, circle packing and variable distribution of frustums 

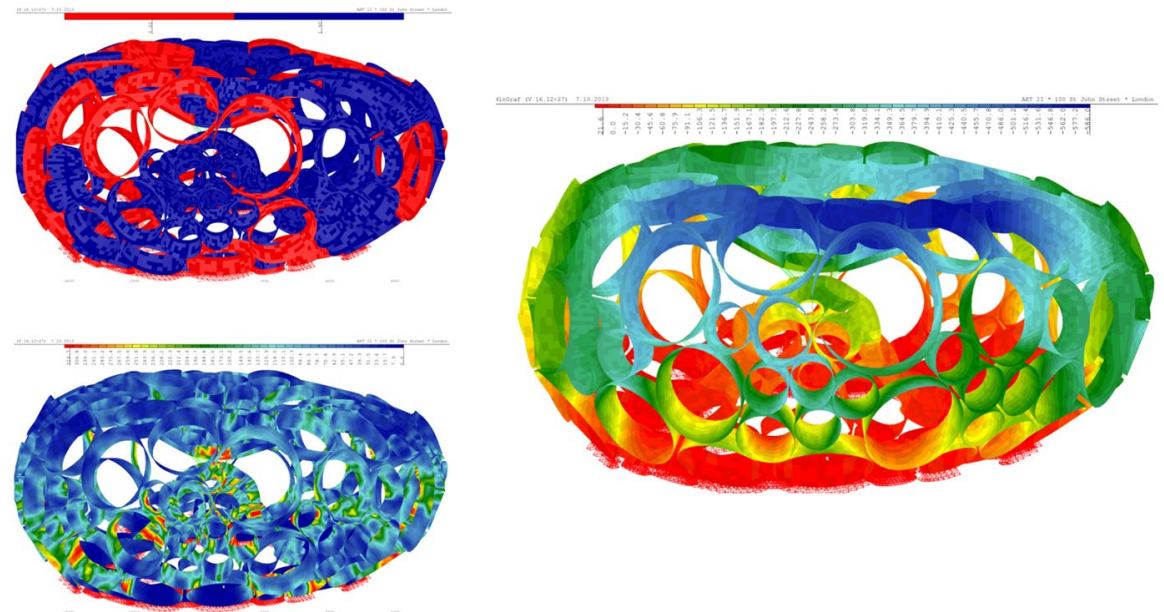

Fig. 6 FEA results, gauge distribution, stresses and displacements

for the frustums boundaries, their associated gauge and their bolt connection holes setting out (see Fig. 7).

The installation was entirely fabricated and installed by Professor DeWitt and a number of local artists, students and alumni. The installation process required the pre-bolting of several circles together off site, and then lifting entire sections in place using a mobile crane. The installation was controlled by resting those sections onto temporary props in order to maintain the given overall setting out. Over 1 week (under adverse meteorological condition including high wind speeds and heavy snow) the team managed to connect all the 250 circles with over 2500 bolts, and
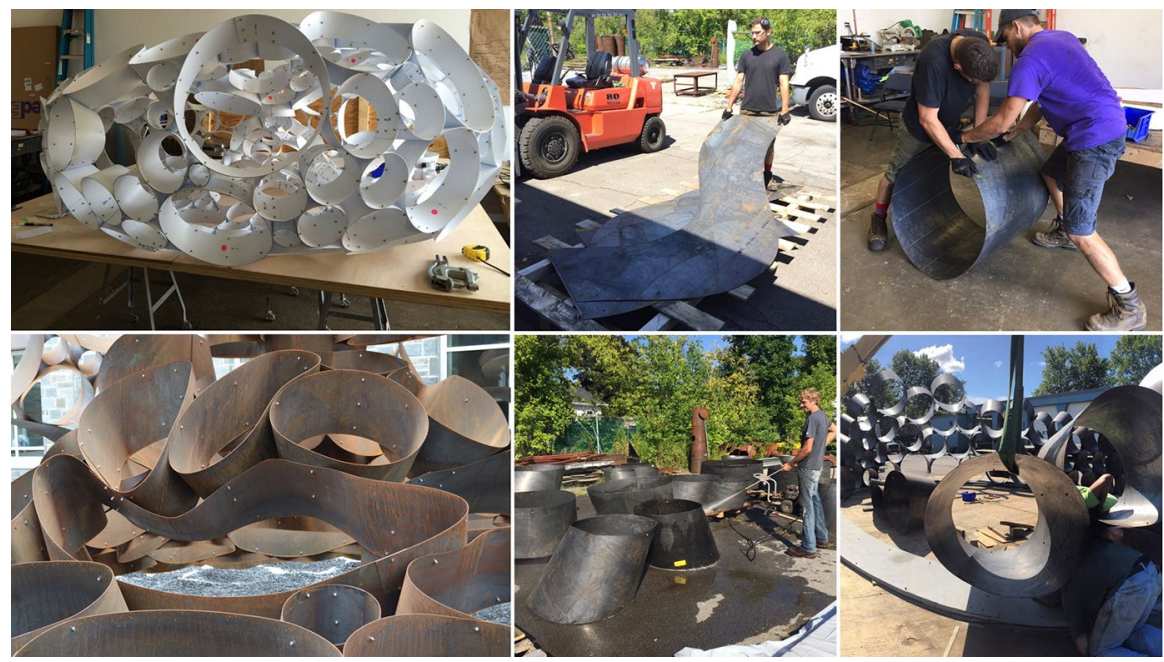

Fig. 7 Fabrication and installation process 
successfully de-propped the installation, which can now be admired in the courtyard of the Colgate University in Hamilton, NY, USA. The dynamic behaviour of the lightweight structure creates a mesmerising effect of undulation, allowing the forces running through the interlocked series of hoops to be evident to the human eye.

\section{'Tangling', Architecture Foundation, London}

Tangling is a self-intersecting structural ribbon, weaving onto itself to create a three-dimensional labyrinthine space from a continuous looping surface. Realised as a timber stress-skin of two cross-laminated layers of ply, separated by a grillage of joists, it's a very simple and economic construction, but nevertheless extremely lightweight and efficient. Commissioned by the Architectural Foundation and installed in their Tooley Street gallery, Tangling completely fills the double-storey space in plan and height, appearing as a gigantic white weaving strip from the street. Inside the gallery, Tangling unfolds as a scroll, revealing a series of miniature models, sketches and projections of Akihisa Hirata's work (see Fig. 8).

The surface was form-found using dynamic relaxation algorithms, also used to simulate the geometry of soap bubbles and other minimal surfaces in nature, from spider webs to coral structures. From the topology of the piece, described in the original architectural model by the architect's studio, the form of the ribbon was 'sprung' into its most natural shape, thus minimising any out of balance forces and enabling the thinnest and lightest structure (see Fig. 9).
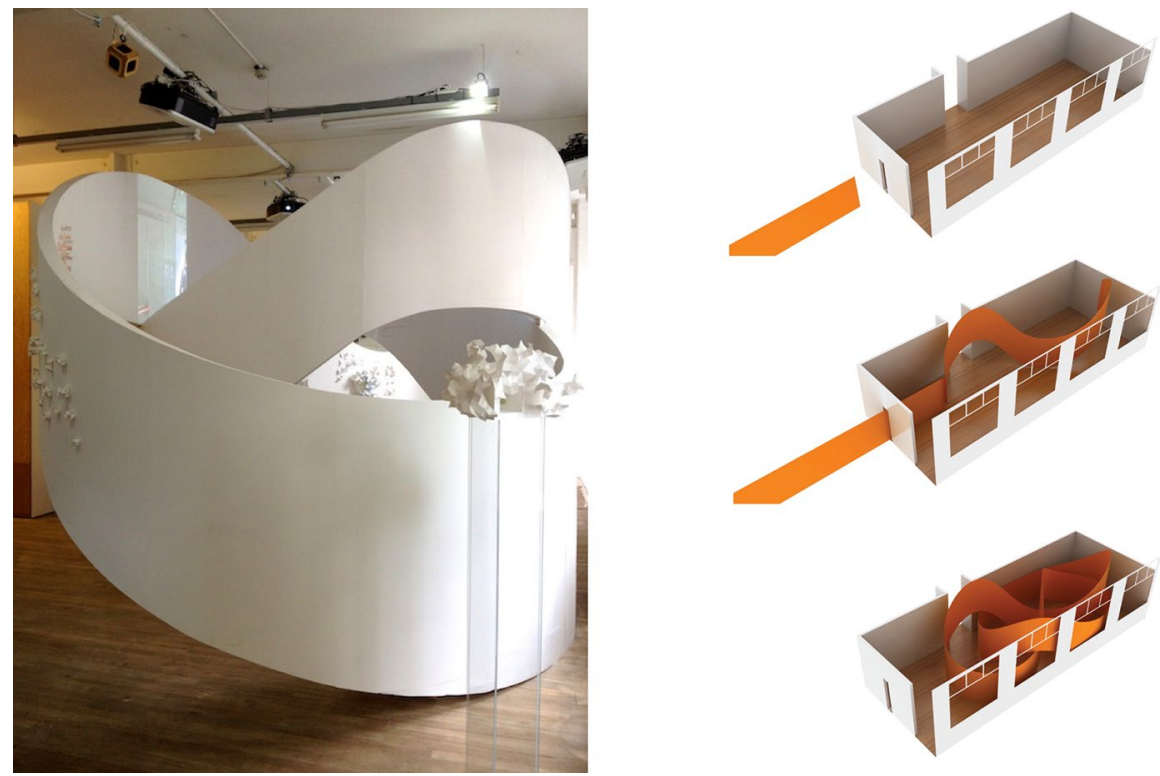

Fig. 8 Tangling, installation and form finding process 


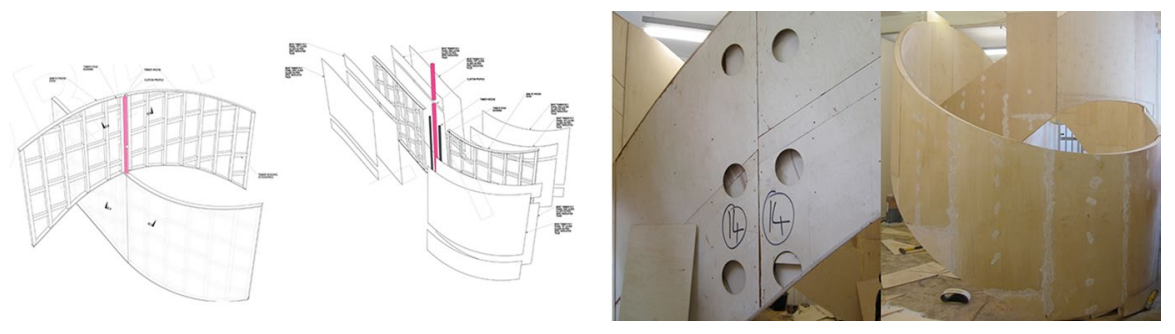

Fig. 9 Detailing, structural stress skin
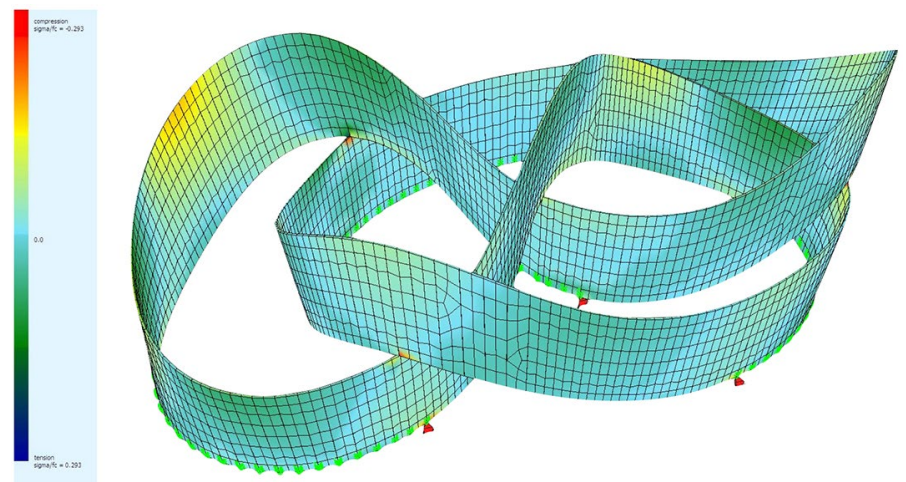

Fig. 10 FEA utilisation results

It was then dissected using a parametric script, written by AKT II, into its unfolded unit-components so that the contractor could fabricate it and assemble it as a kit of parts. The splices through the components were aligned with the areas of minimum stress in order to reduce the connection and overall thickness of the ribbon. This project fully embraced the emergence of a form, which is governed by the physical behaviour of the material that constitutes it and the forces that act upon it. This new aesthetic is based on the orders, rhythms and patterns derived from nature's complex processes. So the undulations of the Tangling ribbon are not casual, they follow specific trajectories and patterns, which make the object intriguing (see Fig. 10).

\section{‘Neuron Pod' at Centre of the Cell, Queen Mary University of London}

The Neuron Pod is a new addition to Centre of the Cell, an award-winning science education centre at Queen Mary University of London's Whitechapel campus, which helps to inspire local children to pursue careers in the sciences and engages with the local community in medical research. To address ever-increasing demand, the Neuron Pod will help increase visitor numbers and provide a multi-functional space for live science shows, hands-on workshops, experiments, debates, films 
and exhibitions. The Pod was designed by aLL Design and engineered by AKT II; fabrication and installation was performed by Littlehampton Welding (see Fig. 11).

Made out of weathering steel, the structure of the Pod consists of an external structural skin stiffened by internal steel ribs. These internal ribs run in both directions to provide stiffness and rigidity to the structural skin. The Pod has a zeppelin-inspired shape, both in plan and elevations, and is supported by three legs. The overall shape presents a curved surface; as the name suggests, it resembles the central part of a neuron, comprising the nucleus, the soma and the axon hillock, while the dendrites are shaped in the form of numerous spikes scattered along the external surface of the Pod. Constructed by the specialist steel fabricator following a process similar to the construction of a ship hull, the structure consists of an $8 \mathrm{~mm}$ developable external plate welded on an internal series of vertical and longitudinal steel ribs.

This system was optimised in the early stages of design in order to minimise plate thickness and maximise the spacing of the internal ribs, which benefited the fabrication time. A detailed structural analysis model helped with understanding the critical aspects of plate buckling and how the compression forces travelling through the steel plates-originating from the bending actionstereotomicended influencing the final plate thickness chosen for the fabrication. Part of the thickness was also considered as sacrificial, as the Pod is intended to be left without protective paint to maintain the weathered steel look. These articulated solids were constructed by precise laser-cut flat metal sheets, curved in place using the vertical ribs as an 'embedded structural formwork'. For this reason, respecting tolerances, understanding the material behaviour when cold formed, and controlling the stresses within the plate once welded (therefore increasing the local temperature) was crucial to maintaining the overall predicted stresses in the structure, compatible with the

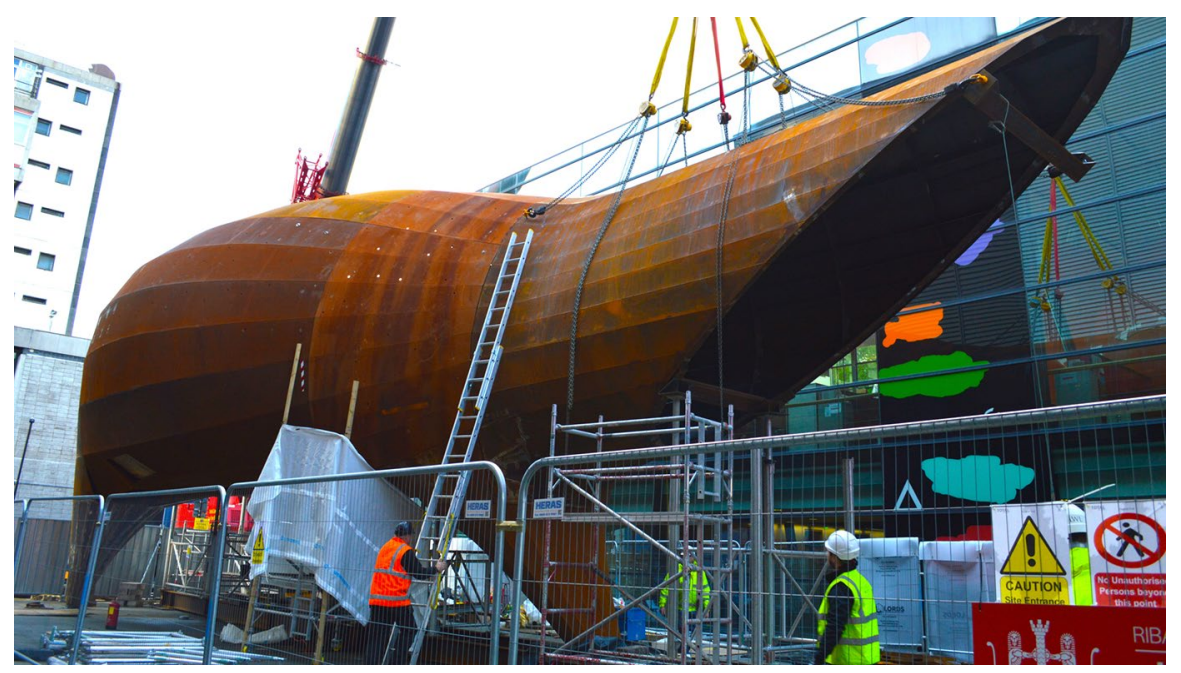

Fig. 11 Neuron pod during installation 

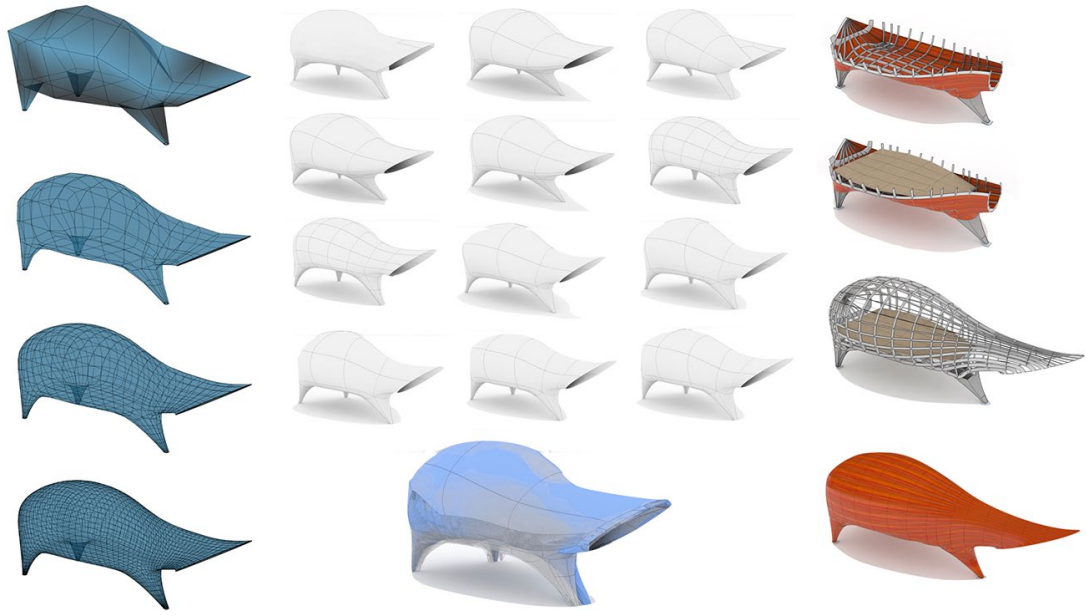

Fig. 12 Form generation and revisions
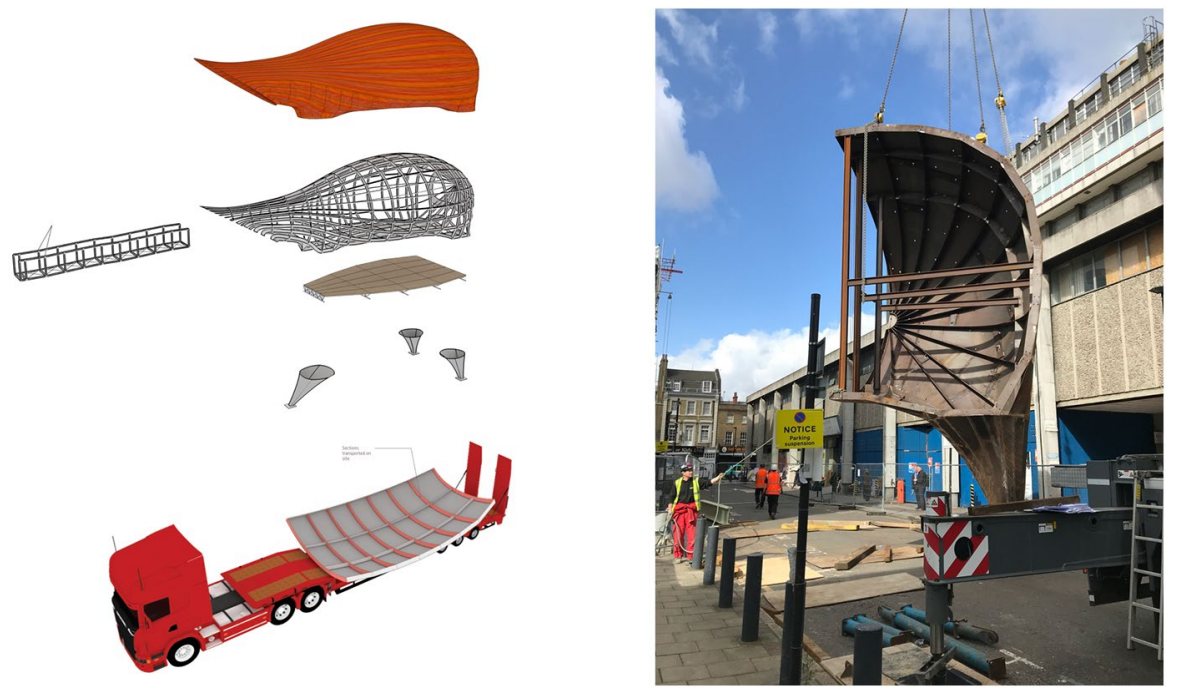

Fig. 13 Diagram of structural components and installation

design. The overall depth of the ribs is a function of the deflection; this system is capable of performing at smaller depths than conventional steel beams.

This project was designed, analysed and produced using a bespoke workflow to generate the surface (see Fig. 12), implement the structural ribbing and splicing following the overall stress patterns, and produce a full set of construction drawings designed to achieve high levels of precision in the final fabricated form. The smooth surface obtained with the AKT II algorithm was envisaged as a starting point. A subsequent step was to study a subdivision pattern to discretise 

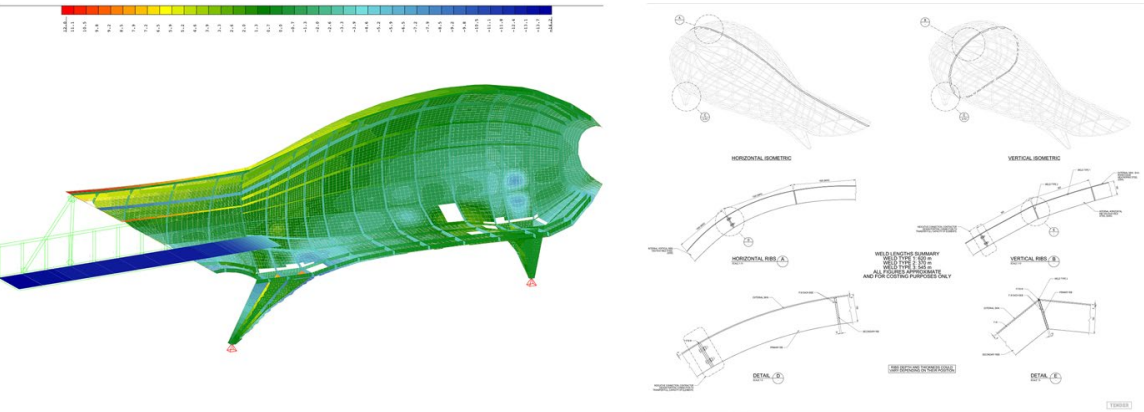

Fig. 14 FEA results and welding diagrams

the overall shape in developable strips. This step allowed a quick generation of various options for the ribs, and once the loading criteria were set, it allowed fine-tuning of the pattern to balance the target of limiting the self-weight (and therefore material) with the need of strength and stiffness. The final result took into consideration this approach and combined it to the fabrication splicing limitations in order to arrive at the final result, which optimised the location of the structure for both performance and fabricability (see Fig. 13). The same digital model generated from the output of the workflow was used by the fabricator to cut, assemble, weld and finalise the final pieces, which were then loaded onto trucks and delivered to their final location (see Fig. 14). The structure was installed following a specific sequence: the various parts were temporarily bolted together in order to minimise the temporary props required on site, and once the jigsaw was complete, the bolted interfaces were welded to create the final structural continuity required, allowing the temporary props to be removed.

\section{Conclusions}

Søren Kierkegaard once wrote: "Life can only be understood backwards; but it must be lived forwards".

Stereotomic techniques were supported by the advances in geometry and mathematics of the time, with every cut performed in the stone joints embedding form, ornament, structural and material performance, and fabrication tolerances. This was also made possible by the use of new ways to describe the 3D objects in a drawing and the enhanced skills of the stone masons that reached their apex concurrently. There is an evident parallelism between traditional stereotomy theory and practice, and the new techniques developing and described in the previous projects. Stereotomy in its modern applications can inform new typologies of structures that integrate technology and can address many of the aesthetic, economic and environmental challenges the AEC industry is facing. 


\section{References}

Block, Philippe, Tom Van Mele, Matthias Rippmann and Noelle Paulson. 2017. Beyond Bending: Reimagining Compression Shells. Munich: DETAIL.

Clifford, Brandon and Wes McGee. 2014. La Voûte de LeFevre: a variable-volume compression-only vault. In: Fabricate: Negotiating Design and Making, eds. Fabio Gramazio, Matthias Kohler and Silke Langenber, 146-153. Zürich: gta Verlag.

Clifford, Brandon, McGee, Wes and James Durham. 2016. Round Room. In: ACADIA Posthuman Frontiers: Data, Designers and Cognitive Machines, Projects Catalog 2016, 190-195. https://stati c1.squarespace.com/static/5307a330e4b06dde7ef78d6f/t/58b2f342579fb3b2016d9983/1488122696 159/2016_190_RoundRoom.pdf. Accessed 10 Jan. 2018.

Colella, Micaela. 2017. Structures, Algorithms and Stone/Timber Prototypes. Nexus Network Journal 19(1): 209-215. https://doi.org/10.1007/s00004-016-0310-Z

Diles, Justin. 2018. Lightweight Stereotomy with Glass-Fiber Reinforced Plastic. Nexus Network Journal 20(2). https://doi.org/10.1007/s00004-018-0376-X

Fallacara, Giuseppe. 2007. Verso una progettazione stereotomica. Nozioni di Stereotomia, Stereotomia digitale e trasformazioni topologiche: ragionamenti intorno alla costruzione della forma. Rome: Aracne Editrice.

Fallacara, Giuseppe. 2012. Stereotomy: Stone Architecture and New Research. Paris: Presses des Ponts et Chaussées.

Fallacara, Giuseppe and Maurizio Barberio. 2017. Parametric morphogenesis, robotic fabrication \& construction of novel stereotomic hypar morphologies: Hypar Gate, Hypar Wall and Hypar Vault. In: Handbook of Research on Form and Morphogenesis in Modern Architectural Contexts, ed. D'Uva Domenico, 329-353. Hershey: IGI Global.

Fallacara, Giuseppe and Maurizio Barberio. 2018. An Unfinished Manifesto for Stereotomy 2.0. Nexus Network Journal 20(2). In press.

Kara, Hanif and Bosia, Daniel. 2016. Design Engineering Refocused. John Wiley \& Sons: London. https ://doi.org/10.1002/9781119164838

McGee, Wes, Feringa, Jelle and Asbjørn Søndergaard. 2013. Processes for an Architecture of Volume. In: Rob|Arch 2012: Robotic Fabrication in Architecture, Art and Design, ed. Sigrid Brell-Cokcan and Johannes Braumann, 62-71. Vienna: Springer-Verlag Wien.

Merritt, Tim R. and Pedro de Azambuja Varela. 2014. Designing And Building The Cork Vault: Reflections On A Two-Week Digital Fabrication Workshop. In: DesignEd Asia, 1-11. http://www. designedasia.com/2014/Full_Papers/2014/24_Designing\%20and\%20building\%20the\%20cork\%20 vault.pdf. Accessed 10 Jan. 2018.

Varela de Azambuja, Pedro and José Pedro Sousa. 2018. Variable Casting of Voussoirs for a Stereotomic Shell. Nexus Network Journal 20(2). In print.

Edoardo Tibuzzi is a designer and researcher based in London (UK). He currently serves as an Associate Director at AKTII, where he leads its Computational Design team (p.art ${ }^{\circledR}$ ). He graduated from the Civil Engineering Faculty of Rome University in 2005 presenting a project of structural restoration of a 15th century Roman Palazzo (Palazzetto Cenci). Subsequently to his degree he moved to London where he developed a profound interest for parametric and computational design. Uncertainty modeling, quantification and mitigation, multi-scale modeling of structures and multidisciplinary design optimization are part of his daily research. In 2007 he joined Adams Kara Taylor (now AKTII) and shortly after became member of its parametric applied research unit (p.art ${ }^{\circledR}$ ). During his work at AKTII, he has completed various projects, in particular the UK Pavilion (Shanghai 2010 Expo's), BMW Pavilion and Coca-Cola Beat-box (London 2012 Olympic Park), Akihisa Hirata's Tangling exhibition (The architecture Foundation, 2012), Birmingham New Street Station Re-development (Birmingham 20092013), Bloomberg's London Headquarter (2012-now), BIG's Serpentine Pavillion, to name a few. He collaborated with KTH University in Stockholm, UCL in London and Tor Vergata University in Rome, running various lectures and workshops. He co-led a cluster in 2013 Smart Geometry, and co-authored the book: Design Engineering RE-Focused published in 2016 by Wiley. 